\title{
Assessment of ventilation inhomogeneity during mechanical ventilation using a rapid-response oxygen sensor-based oxygen washout method
}

\author{
Ido G Bikker ${ }^{1,3}$, Wim Holland ${ }^{2}$, Patricia Specht ${ }^{3}$, Can Ince ${ }^{1}$ and Diederik Gommers ${ }^{1 *}$
}

\author{
* Correspondence: \\ d.gommers@erasmusmc.nl \\ ${ }^{1}$ Department of Intensive Care, \\ Erasmus Medical Center, PO Box \\ 2040, Rotterdam 3000 CA, the \\ Netherlands \\ Full list of author information is \\ available at the end of the article
}

\begin{abstract}
Purpose: Ventilatory inhomogeneity indexes in critically ill mechanically ventilated patients could be of importance to optimize ventilator settings in order to reduce additional lung injury. The present study compared six inhomogeneity indexes calculated from the oxygen washout curves provided by the rapid oxygen sensor of the LUFU end-expiratory lung volume measurement system.

Methods: Inhomogeneity was tested in a porcine model before and after induction of acute lung injury (ALI) at four different levels of positive end-expiratory pressure (PEEP; 15, 10, 5 and $\mathrm{O} \mathrm{cm} \mathrm{H}_{2} \mathrm{O}$ ). The following indexes were assessed: lung clearance index $(\mathrm{LCl})$, mixing ratio, Becklake index, multiple breath alveolar mixing inefficiency, moment ratio and pulmonary clearance delay.

Results: $\mathrm{LCl}$, mixing ratio, Becklake index and moment ratio were comparable with previous reported values and showed acceptable variation coefficients at baseline with and without ALI. Moment ratio had the highest precision, as calculated by the variation coefficients. $\mathrm{LCl}$, Becklake index and moment ratio showed comparable increases in inhomogeneity during decremental PEEP steps before and after ALI.

Conclusions: The advantage of the method we introduce is the combined measurement of end-expiratory lung volume (EELV) and inhomogeneity of lung ventilation with the LUFU fast-response medical-grade oxygen sensor, without the need for external tracer gases. This can be combined with conventional breathing systems. The moment ratio and $\mathrm{LCl}$ index appeared to be the most favourable for integration with oxygen washout curves as judged by high precision and agreement with previous reported findings. Studies are under way to evaluate the indexes in critically ill patients.
\end{abstract}

Keywords: Lung; Respiratory monitoring; Ventilatory inhomogeneity; Positivepressure respiration

\section{Background}

Although mechanical ventilation is critical for the survival of most patients with respiratory failure, it can also induce lung damage and may even be the primary factor in lung injury [1].

In 1970, Mead et al. estimated that forces acting on lung tissue might be 4.5 times higher when lungs are inhomogeneously ventilated [2]. This was confirmed in an experimental work using tomographic microscopy, which generates detailed three-dimensional

\section{Springer}

(c) 2014 Bikker et al.; licensee Springer. This is an Open Access article distributed under the terms of the Creative Commons Attribution License (http://creativecommons.org/licenses/by/2.0), which permits unrestricted use, distribution, and reproduction in any medium, provided the original work is properly credited. 
alveolar geometries [3]. This inhomogeneity raises stress and increases the risk to develop ventilator-induced lung injury (VILI). Therefore, the use of an inhomogeneity index as a target for ventilation strategies would be very beneficial in this context.

Although inhomogeneity indexes are often used in pulmonary function labs and improve after application of positive end-expiratory pressure (PEEP) in paediatric anaesthesia, use in the intensive care unit (ICU) is limited by the need of specialized equipment and tracer gases [4,5]. Huygen et al. [6,7] and Gültuna et al. [8] worked on the development of inhomogeneity indexes and indicator gas injectors based on SF6 for critically ill patients, but implementation remained difficult due to the need of specialized equipment and gas containers at the bedside [6,7]. The availability of a routine method to quantify inhomogeneous alveolar ventilation at the bedside is expected to help to optimize ventilator settings in individual patients to achieve optimal gas exchange. Recently, methods were introduced to measure end-expiratory lung volume (EELV) with an ICU ventilator based on an oxygen washout curve $[9,10]$. In the LUFU system developed by Weismann et al., oxygen concentration is measured with a diverting oxygen analyser with response time $<200 \mathrm{~ms}$. This method offered the possibility to develop an index of ventilatory inhomogeneity which we believe will be of great potential use in combination with EELV measurement to optimize ventilatory settings at the bedside.

The aim of this study was to develop a bedside-available computer program to compare six well-established indexes of ventilatory inhomogeneity calculated from the oxygen washout/washin curve of the LUFU system [11]. The system was evaluated for reproducibility and agreement with historical reference values in a porcine model before and after induction of acute lung injury (ALI), at four different levels of PEEP.

\section{Methods}

The study was approved by the local animal experimental committee and was in accordance with the National Guidelines for Animal Care and Handling (permit number 142-08-01).

\section{Description of the indexes of ventilatory inhomogeneity}

Ventilatory inhomogeneity is calculated from an oxygen washout procedure performed with the LUFU system (Dräger Medical, Lubeck, Germany). This system was developed to measure EELV based on oxygen washout and has been described earlier [12]. Briefly, $\mathrm{O}_{2}$ is measured with a side stream sensor, and airway flow is retrieved from the EVITA XL ventilator (Dräger Medical, Lubeck, Germany). A step change in the $\mathrm{O}_{2}$ concentration is induced by changing the $\mathrm{FiO}_{2}$. Oxygen concentration and flow are integrated in the LUFU system, and the EELV is calculated from the washout or washin procedure.

The indexes were calculated as described in the article by Larsson et al. [11] and are shown in Figure 1. All indexes were calculated with a computer model programmed in MATLAB (MathWorks, Natick, MA, USA). Apparatus dead space was always subtracted from the tidal volume measurement when cumulative ventilation was calculated. 'Volume turnover' (TO) is defined as the cumulative expired volume divided by EELV. Some indexes are designed to be less dependent on tidal volume, dead space and lung volumes by comparing the measured inhomogeneity to inhomogeneity in an ideal lung. Ideal number of breaths and ideal volume turnovers refer to the washout in the simulated lung, which has the same EELV, dead space and tidal volume as the patient, but with uniform alveolar gas. 


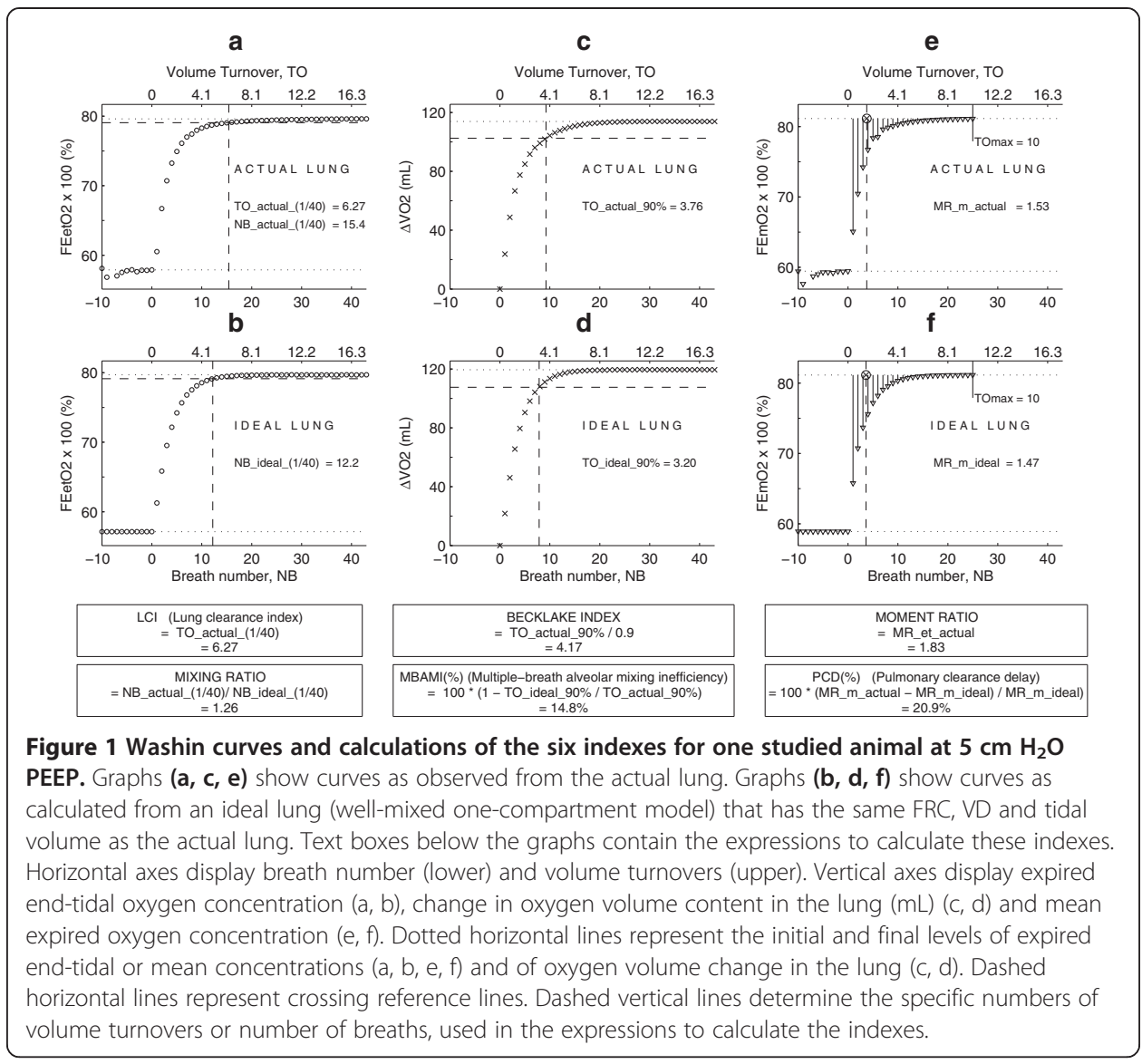

Lung clearance index (LCI) equals the number of observed volume turnovers required to reach the final end-tidal oxygen concentration within a 1/40th part of the total oxygen shift (difference between final and initial concentration levels; see Figure 1a). In Figure 1, the vertical dashed line is drawn through the intersection point of the horizontal crossing level line with the washin curve. Its upper $x$-axis coordinate value determines the needed number as TO_actual_(1/40) [13].

Mixing ratio is the ratio between the observed and the ideal number of breaths required to reach the final end-tidal oxygen concentration within a 1/40th part of the total oxygen concentration shift (see Figure 1a,b). The vertical dashed lines determine these numbers as NB_actual_(1/40) and NB_ideal_(1/40), respectively [14].

Becklake index is the number of volume turnovers required to wash $90 \%$ of the ultimate total increase of oxygen content in the lung, divided by 0.9 (see Figure 1c). The vertical dashed line is drawn through the interception point of the horizontal crossing level line with the washin curve. Its upper $x$-axis coordinate value determines the needed number as TO_actual_90\% [15].

Multiple-breath alveolar mixing inefficiency (MBAMI) is defined as $100 \times(1-$ Volume turnover ideal/Volume turnover actual), where the number of volume turnovers refers to how many turnovers are required to wash $90 \%$ of functional residual capacity (FRC) free of tracer gas (Figure 1c,d) [16]. 
Moment ratio is designed to summarize the whole washout curve using moment analysis; it equals the mean residence time. It is the ratio between the first $\left(\mu_{1}\right)$ and zeroth $\left(\mu_{0}\right)$ moments of the washout curve [17]:

$$
\mu_{0}=\sum_{k=1}^{N} Y(k)\left[\eta_{k}-\eta_{k-1}\right]
$$

and

$$
\mu_{1}=\sum_{k=1}^{N} \eta_{k} Y(k)\left[\eta_{k}-\eta_{k-1}\right]
$$

where $k$ is the number of breaths, $\eta$ is the number of volume turnovers at breath $k, N$ is the number of the washout breaths at which $\eta_{k}$ exceeds a preselected value (10, as mostly used), and $Y(k)$ is the end-tidal tracer gas concentration at breath $k$ minus by the concentration at the end of washin. We used the same calculation as Larsson et al. who started the summations with the first washout breath $(k=1)$. The calculated value of MR_et_actual is shown in Figure 1 upper right text box [11]. A mechanical analogon may help to understand the concept of moment ratio. Consider a mechanical balance on which a weightless beam is attached a series of weights proportional to the length of the line pieces and positioned along the beam as suggested in Figure 1e,f. The horizontal position of the pivotal point (encircled cross mark) where the beam balances determines the moment ratio.

Pulmonary clearance delay (PCD; \%) is calculated as the alternative method proposed by Larsson et al., based on moment ratio: PCD $(\%)=100 \times$ (Actual moment ratio - Ideal moment ratio)/Ideal moment ratio [11]. This way of expressing PCD has the advantage of being easily adapted to automatic calculation. Contrary to Larsson et al., we truncated the summation process at $\mathrm{TO}=10$ (see Figure $1 \mathrm{e}, \mathrm{f}$ ). The values involved in the summation are shown as vertical solid line pieces. The involved moment ratio's named MR_m_actual and MR_m_ideal for the actual and the ideal lung, respectively, are indicated by the drawn dashed lines.

The indexes were evaluated for integration with oxygen-based washout and automated calculation; precision was calculated as the variation coefficient. Also, values were compared with previously reported findings, also used by Larsson et al. [11].

\section{LUFU system}

The LUFU system to measure EELV was developed and described in great detail by Weismann et al. [12]. Briefly, this system measures only oxygen with a small diverting oxygen analyser (response time $<200 \mathrm{~ms}$ ). Currently, this system has to be connected to a Dräger ICU ventilator; in the current study, we used an Evita XL ventilator. Measurements are started by manually changing the inspired oxygen fraction with a change of at least $10 \%$. The LUFU system then calculates the EELV from the washout/in curve, without the need for external tracer gases. Because only suitable side stream sensors are available, the resulting problem of corrupted synchronicity between flow and gas concentration measurement was solved by the use of an ingenious physical/mathematical model of the pneumatical circuit of the analyser $[12,18]$. Also, the washout curve has to be meticulously corrected for oxygen consumption; the oxygen consumption is always calculated during 
the ten breaths before each measurement. As described by Weismann et al., measurements should be performed during steady state, with a stable cardiac output.

\section{Animal preparation}

Seven healthy female cross-bred Yorkshire/Landrace pigs (28 to $31 \pm 1.2 \mathrm{~kg}$ ) were studied. After induction, the pigs were placed in the supine position on a thermo-controlled operation table. Anaesthesia and analgesia were maintained with an intravenous infusion with a combination of midazolam (1 to $1.7 \mathrm{mg} / \mathrm{kg} / \mathrm{h}$ ) and sufentanil $(0.01$ to $0.02 \mathrm{mg} / \mathrm{kg} / \mathrm{h}$, Sufenta Forte $0.05 \mathrm{mg} / \mathrm{mL}$, Janssen-Cilag BV, Tilburg, the Netherlands). Muscle relaxation was obtained with an infusion of pancuronium bromide $(0.17$ to $0.33 \mathrm{mg} / \mathrm{kg} / \mathrm{h}$, Pavulon $2 \mathrm{mg} / \mathrm{mL}$, NV Organon, Oss, the Netherlands). After tracheotomy, the pigs were connected to an EVITA XL ventilator.

In addition, an arterial catheter was inserted through the right carotid artery and a pulmonary artery thermodilution catheter (7.5 Fr, Edwards Life Sciences, Irvine, CA, USA) through the right jugular vein in the pulmonary artery. A catheter was also placed in the urinary bladder to avoid urine retention. Ventilatory data and volume-based capnography were sampled continuously during the experiment (NICO, Novametrix, Wallingford, CT, USA). Mechanical ventilation and washin and washout procedures to obtain the oxygen curves for lung volume and inhomogeneity index calculations were performed with the EVITA XL ventilator in a volume-controlled mode. Tidal volume was set at $8 \mathrm{~mL} / \mathrm{kg}$, respiratory rate was adjusted to a $\mathrm{PaCO}_{2}$ of 4.5 to $6.0 \mathrm{kPa}$, the inspiratory to expiratory ratio was $1: 2$ and PEEP was $5 \mathrm{~cm} \mathrm{H}_{2} \mathrm{O}$.

\section{Experimental protocol}

After a stabilization period of $30 \mathrm{~min}$, baseline values were recorded, and the pre-lung injury measurements were performed. Lung volume was measured with the LUFU system and was performed in quadruplicate (four washin and four washout) by changing the $\mathrm{FiO}_{2}$ from 0.8 to 0.6 and from 0.6 to 0.8 . Washin and washout procedures were started immediately after the end of the previous procedure. After the repeatability measurements at baseline $\left(5 \mathrm{~cm} \mathrm{H}_{2} \mathrm{O}\right.$ PEEP), inhomogeneity was measured at four decremental PEEP levels (15, 10, 5 and $0 \mathrm{~cm} \mathrm{H}_{2} \mathrm{O}$ PEEP). Single measurements (one washin and one washout) were performed after a steady state of $15 \mathrm{~min}$ at the end of each PEEP step.

Severe ALI was induced by injection of oleic acid $\left(0.13 \pm 0.04 \mathrm{~mL} / \mathrm{kg}, \mathrm{C}_{18} \mathrm{H}_{34} \mathrm{O}_{2}\right.$, Boom BV, Meppel, the Netherlands) into the right atrium with the PEEP set at $2 \mathrm{~cm}$ $\mathrm{H}_{2} \mathrm{O}$. Each animal received a bolus of $0.1 \mathrm{~mL} / \mathrm{kg}$ injected over 20 min to obtain a $\mathrm{PaO}_{2}$ below $10 \mathrm{kPa}$; if necessary, additional injections were given to reach this target [19]. After a steady state of at least $1.5 \mathrm{~h}$ after the induction of ALI, PEEP was increased to $5 \mathrm{~cm} \mathrm{H}_{2} \mathrm{O}$, and baseline measurements were repeated. Thereafter, the same PEEP trial was performed as described above.

\section{Statistical analysis}

Statistical analysis was performed with the GraphPad software package (GraphPad Software Inc., San Diego, CA, USA). Results are expressed as mean \pm standard deviation (SD) for normally distributed data and median \pm interquartile range (IQR) for abnormally distributed data. The Shapiro-Wilk normality test was used to evaluate the distribution of all 
data. Changes in the inhomogeneity indexes, and hemodynamic and ventilatory parameters before and after induction of ALI, were evaluated with the Wilcoxon matched-pairs test. The Wilcoxon matched pairs-test was also used to evaluate change in the inhomogeneity indexes between PEEP steps during the decremental PEEP trial.

\section{Results}

Oleic acid infusion significantly decreased EELV, arterial oxygenation and respiratory compliance (Table 1). Venous admixture and the ratio of alveolar dead space volume divided by alveolar tidal volume (VDalv/VTalv) were both increased by the induction of ALI, indicating an increased ventilation-perfusion mismatch (Table 1). Effects of the different PEEP steps on EELV, $\mathrm{PaO}_{2} / \mathrm{FiO}_{2}$ (PF) ratio and dynamic compliance are shown in Table 2 and Figure 2.

The calculated six ventilation inhomogeneity indexes during baseline are shown in Table 3. The mean variation coefficients, as a measure of repeatability, are also presented (Table 3). LCI, mixing ratio, Becklake index and moment ratio were comparable with historical reference values and showed acceptable mean variation coefficients (Table 3). MBAMI and PCD were not comparable with historical reference values and showed very low repeatability (mean variation coefficient 33.5 and 29.3, respectively). PCD showed no correlation between washout and corresponding washin measurements. After induction of ALI, LCI, Becklake index, MBAMI and moment ratio significantly increased (Table 3 ). The slope of phase 3 of the volume-based capnogram was also significantly increased after induction of ALI (Table 3).

The inhomogeneity indexes at the different PEEP levels are shown in Table 2. Before ALI, LCI and moment ratio significantly increased after lowering PEEP from 10 to $5 \mathrm{~cm} \mathrm{H}_{2} \mathrm{O}$ and from 5 to 0 (Table 2). Becklake index and PCD significantly increased after lowering PEEP from 5 to 0 before ALI (Table 2). The mixing ratio showed no response to PEEP changes before the induction of ALI. After induction of ALI, almost all indexes (except mixing ratio) increased significantly after lowering PEEP from 15 to $10 \mathrm{~cm} \mathrm{H}_{2} \mathrm{O}$, and all indexes increased significantly after lowering PEEP from 10 to $5 \mathrm{~cm} \mathrm{H}_{2} \mathrm{O}$ (Table 2). At ZEEP, no significant differences were found due to a lower sample size as we were not able to measure reliably with the extensive lung injury

Table 1 Data on hemodynamic and ventilatory parameters before and after induction of lung injury during baseline

\begin{tabular}{lcc}
\hline & $\begin{array}{c}\text { Before acute lung injury } \\
(\boldsymbol{n}=\mathbf{7})\end{array}$ & $\begin{array}{c}\text { After acute lung injury } \\
(\boldsymbol{n}=\mathbf{7})\end{array}$ \\
\hline Heart rate $(\mathrm{bpm})$ & $84(15)$ & $126(29)^{*}$ \\
$\mathrm{MAP}(\mathrm{mmHg})$ & $87(8)$ & $88(10)$ \\
$\mathrm{PaCO}_{2}(\mathrm{kPa})$ & $4.7(0.5)$ & $6.4(0.5)^{*}$ \\
$\mathrm{PaO}_{2}(\mathrm{kPa})$ & $54.2(4.0)$ & $8.0(4.3)^{*}$ \\
Shunt $(\%)$ & $10(3)$ & $52(19)^{*}$ \\
VD alveolar/NT alveolar $(\%)$ & $28(5)$ & $44(7)^{*}$ \\
Compliance $\left(\mathrm{mL} / \mathrm{cm} \mathrm{H} \mathrm{H}_{2} \mathrm{O}\right)$ & $22(4)$ & $10(2)^{*}$ \\
EELV $(\mathrm{L})$ & $0.65(0.12)$ & $0.21(0.02)^{*}$ \\
Lactate $(\mathrm{mmol} / \mathrm{L})$ & $0.9(0.4)$ & $0.9(0.3)$ \\
\hline
\end{tabular}

Data are means \pm SD. ${ }^{*} p<0.05$. 
Table 2 EELV and inhomogeneity index parameters during different levels of PEEP before and after $A$ LI

\begin{tabular}{|c|c|c|c|c|c|c|c|c|}
\hline & \multicolumn{8}{|c|}{ PEEP $\left(\mathrm{cm} \mathrm{H}_{2} \mathrm{O}\right)$} \\
\hline & \multicolumn{4}{|c|}{ Before acute lung injury } & \multicolumn{4}{|c|}{ After acute lung injury } \\
\hline & 15 & 10 & 5 & 0 & 15 & 10 & 5 & $0 a$ \\
\hline$\overline{\mathrm{EELV}}(\mathrm{mL})$ & 1,458 & $1,064^{*}$ & $720^{*}$ & $400^{*}$ & $848^{*}$ & $446^{*}$ & $238^{*}$ & $182^{*}$ \\
\hline Lung clearance index & 6.1 & 6.3 & $6.4^{*}$ & $7.3^{*}$ & 7.2 & $9.8^{*}$ & $12.9^{*}$ & 19.7 \\
\hline Mixing ratio & 1.2 & 1.2 & 1.2 & 1.1 & 1.2 & 1.3 & $1.5^{*}$ & 1.4 \\
\hline Becklake index & 4.2 & 4.2 & 4.5 & $5.6^{*}$ & 4.9 & $6.7^{*}$ & $11.7^{*}$ & 19.3 \\
\hline MBAMI (\%) & 13.6 & 12.9 & 14.8 & 20.6 & 15.6 & $25.8^{*}$ & $45.2^{*}$ & 39.5 \\
\hline Moment ratio & 1.6 & 1.7 & $1.7^{*}$ & $2.0^{*}$ & 2.0 & $2.4^{*}$ & $2.9^{*}$ & 3.9 \\
\hline Pulmonary clearance delay (\%) & 14.7 & 13.0 & 10.6 & $10.0^{*}$ & 15.9 & 17.4 & 20.2 & 10.3 \\
\hline
\end{tabular}

Values are expressed as means. ${ }^{*} p<0.05$, significance compared to PEEP 15 (Wilcoxon matched-pairs test), $a ; n=6$ (unable to perform measurements in two piglets).

caused by the oleic acid. MBAMI and PCD values may not be reliable due to the low repeatability as shown in the baseline measurements.

\section{Discussion}

This paper describes the assessment and integration of a ventilatory inhomogeneity index based on a rapid oxygen sensor incorporated into LUFU equipment without the need for external tracer gases. The moment ratio and LCI index appeared to be the most favourable for integration with oxygen washout curves. To our knowledge, this is the first study to describe indexes of alveolar inhomogeneity measured by medicalgrade oxygen sensors and conventional breathing systems and applicable during mechanical ventilation.

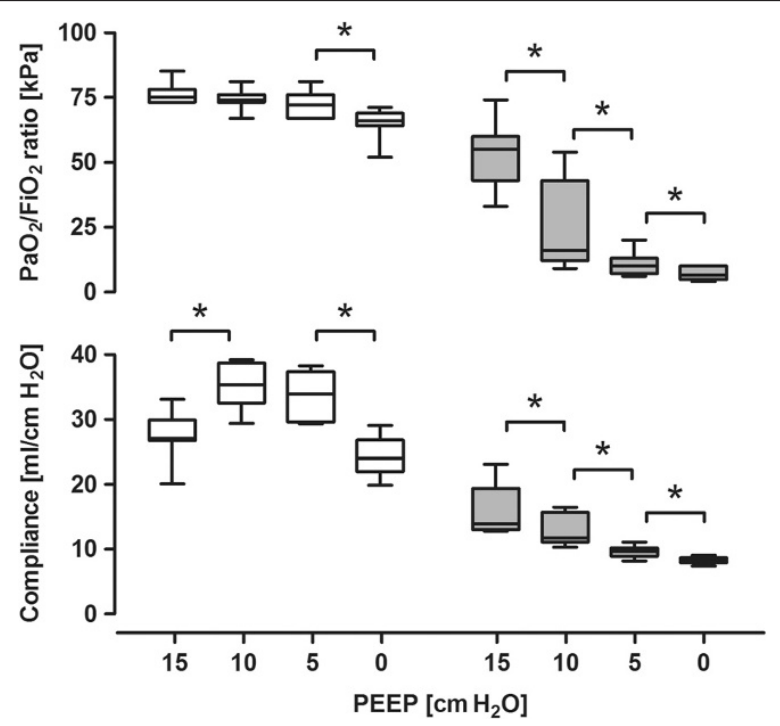

Figure $2 \mathrm{PaO}_{2} / \mathrm{FiO}_{2}$ ratio and dynamic compliance at the four decremental steps in PEEP levels. Before (open bars) and after (grey bars) the induction of acute lung injury in a porcine model. Data are presented as box-and-whisker plots (5 to 25 -median - $75 \%$ to $95 \%$ ). Asterisks indicate a significant difference between the PEEP levels $(p<0.05)$ 
Table 3 EELV and inhomogeneity index parameters before and after induction of lung injury during baseline

\begin{tabular}{lcccc}
\hline & $\begin{array}{c}\text { Before acute } \\
\text { lung injury } \\
(\boldsymbol{n}=\mathbf{7})\end{array}$ & $\begin{array}{c}\text { After acute } \\
\text { lung injury } \\
(\boldsymbol{n}=\mathbf{7})\end{array}$ & $\begin{array}{c}\text { Mean } \\
\text { variation coefficient \% }\end{array}$ & $\begin{array}{c}\mathbf{N}_{\mathbf{2}} \text { washout in normal } \\
\text { subjects [11] }\end{array}$ \\
\hline EELV $(\mathrm{mL})$ & $537(127)$ & $159(55)^{* *}$ & 5.2 & \\
Lung clearance index & $6.7(0.6)$ & $12.7(4.3)^{* *}$ & 10.4 & $7.1(1.3)$ \\
Mixing ratio & $1.2(0.1)$ & $1.3(0.1)$ & 7.8 & $1.57(0.22)$ \\
Becklake index & $4.6(0.5)$ & $11.2(4.5)^{* *}$ & 11.3 & $3.7(0.8)$ \\
MBAMl (\%) & $14.6(6.0)$ & $35.8(19.4)^{*}$ & 33.5 & $25(10)$ \\
Moment ratio & $1.8(0.1)$ & $3.0(0.5)^{*}$ & 5.6 & $2.02(0.14)$ \\
$\begin{array}{l}\text { Pulmonary clearance } \\
\text { delay mean (\%) }\end{array}$ & $11.0(4.2)$ & $13.7(7.8)$ & 29.3 & $31(25)$ \\
$\begin{array}{l}\text { Slope phase 3 capnogram } \\
\text { (kPa/L) }\end{array}$ & $2.1(1.2)$ & $7.0(3.7)^{*}$ & & \\
\hline All & & & & \\
\hline
\end{tabular}

All measurements were performed at $5 \mathrm{~cm} \mathrm{H} \mathrm{H}_{2} \mathrm{O}$ of PEEP. Capnogram values are averaged over 100 successive breaths and increased significantly after induction of ALI. Values are expressed as mean (SD). ${ }^{*} p<0.05 ;{ }^{* *} p<0.01$ (Wilcoxon matched-pairs test)

\section{Technical considerations}

Although numerous indexes have been proposed, all require specialized equipment (e.g. mass spectrometer) and/or tracer gases (e.g. SF6, helium) [20]. To date, unfortunately, no ventilatory inhomogeneity index has been implemented for routine use in adult critically ill patients. Inhomogeneity can be determined based on single-breath or multiple-breath washout [21]. Single-breath washout is clinically available with exhaled volumetric capnography. The upslope of the third phase of this capnogram can be used as a measure of ventilatory inhomogeneity. However, a breath from vital capacity to residual volume should be used to analyse the accessible lung; during normal tidal breathing, only inhomogeneity of this ventilated volume is shown. Also, the contribution of peripheral airways is unknown. The advantage of a multiple-breath washout technique includes the evaluation of both convention- and diffusion-dependent inhomogeneity and the possibility of application during normal tidal breathing without patient cooperation. To obtain a multiple-breath washout index without the use of an insoluble external tracer gas, nitrogen and oxygen can be used as naturally available gases. Most indexes use nitrogen but require a mass spectrometer for a precise washout curve [11,20,22]. Oxygen washout without advanced equipment is limited by slow medical-grade oxygen sensors and the oxygen consumption correction [23]. The present study uses an improved oxygen sensor integrated in the LUFU system, originally developed and validated for EELV measurements $[9,12]$. Importantly, the LUFU equipment corrects for the changing inspired oxygen fraction, which influences the gas viscosity and thereby flow through the side stream sampling system [12].

Oxygen consumption is a major factor, complicating the use of oxygen as a tracer gas. Weismann et al. developed the LUFU method for EELV measurement-based oxygen washout and previously described the calculations and basic conditions [12]. In short, oxygen transport through the lung membrane is regarded as constant throughout the washin/washout cycle. It is calculated as the oxygen consumption and tissue oxygen. During measurements, patients must be stable, with stable lung perfusion and oxygenation. Absorption is measured during stable conditions at the end of each washin or washout. 
This constant consumption is subtracted from each breath during washin/washout. Under these conditions, oxygen consumption does not influence these conditions.

We also implemented an automated correction for small artefacts of the washout curve in our program. The use of the fast LUFU oxygen sensor can easily lead to small disturbances caused by environmental factors (e.g. vibrations). We observed short negative disturbances in two to three successive breaths. An automated detection was implemented and correction was performed with interpolated values.

\section{Observed effects in the inhomogeneity indexes}

We were able to calculate several inhomogeneity indexes based on oxygen washout with acceptable repeatability. At baseline, LCI, mixing ratio, Becklake index and moment ratio were comparable with historical reference values and showed acceptable variation coefficients. Moment ratio had the lowest variation coefficient, which nearly approached the EELV measurement itself. We were not able to measure inhomogeneity reliably and consistently with the PCD and MBAMI indexes. In the automatically calculated PCD, further analysis even showed no correlation between washin and washout. A possible explanation could be an attempt to compensate indexes for tidal volume, dead space and EELV by comparing the actual measured effects to the ideal effects simulated in an ideal lung (PCD, MBAMI and mixing ratio). This could exaggerate the influence of small artefacts on the calculations. The good reproducibility of the moment ratio may be explained by the underlying principle. It is not dependent on a piece of the washout curve, but rather takes the whole washout curve into account, resulting in a mean number of volume turnovers. LCI and mixing ratio are depending on the point at which the concentration is reduced to 1/40th of the initial concentration. Becklake index and MBAMI are dependent on the point where $90 \%$ of the tracer gas has been washed out and thus are heavily dependent on the tail of the washout curve. The tail of the washout curve may be especially vulnerable to artefacts when using oxygen as a soluble tracer gas.

Induction ventilatory inhomogeneity by oleic acid increases all indexes, with a significant increase in the LCI, Becklake index, MBAMI and moment ratio. This is in agreement with Tsang et al. [24]. In their study, ALI was induced with oleic acid in eight mongrel dogs, and the multiple-breath helium washout technique was used to analyse the development of ventilatory inhomogeneity. Inhomogeneity increased mainly due to an increase in the heterogeneity of tissue compliance in the peripheral airways, airway closure and a decrease in ventilation through collateral channels [24]. We also observed a significant increase in inhomogeneity using the volumetric capnogram over 100 successive breaths at baseline which evaluates breath inhomogeneity [25]. As the heterogeneity in mixing of alveolar units with different time constants increases, the slope of phase 3 of the capnogram will increase.

The LCI, Becklake index and moment ratio showed comparable increases in inhomogeneity during decremental PEEP steps before and after ALI. As shown in Figure 2, the decremental PEEP steps resulted in major reductions in PF ratio and compliance, which suggests an increased ventilation/perfusion mismatch due to inhomogeneous ventilation. The dilution factor (ratio alveolar tidal volume/EELV) could influence these results. If changed by increasing PEEP and thereby EELV, this could delay washout and increase the measured inhomogeneity. In our study, we did not find increased inhomogeneity at higher PEEP. If inhomogeneity indexes were to be used for evaluating the optimal PEEP setting, 
one would not only be interesting during lower PEEP steps where an increased shunt fraction can be expected. Higher PEEP may lead to lung overdistension, resulting in uneven ventilation. In our study, no increase in inhomogeneity was seen in any of the indexes. Whether these indexes are insensitive to overdistention or the used PEEP steps to low is currently unknown.

\section{Which index based on oxygen washout to use in critically ill patients?}

In our study, LCI, mixing ratio, Becklake index and moment ratio showed acceptable precision. Inhomogeneity expressed by moment ratio analysis showed the highest reproducibility, and this index was able to detect the oleic acid-induced inhomogeneity and may be able to detect PEEP-induced changes. With its relatively low dependence on a specific part of the washout curve and thereby relatively insensitive to small artefacts, it may be favourable for oxygen washout. LCI had a slightly higher variation coefficient, but it is currently the most described inhomogeneity index in pulmonary function labs, has even been suggested as the end point for clinical trials [13] and is easy to understand. Comparing the measured values with the ideal values for an ideal lung (as used by MBAMI, PCD and mixing ratio) could theoretically improve results and reliability. In our study, this could not be confirmed; PCD and MBAMI showed low precision.

\section{Limitations}

In the current study, we used a $20 \%$ step change in the inspired oxygen fraction, which should be feasible in most critically ill patients. Only the most seriously ill patients would require the lower step change of $10 \%$. Also, we did not study the impact of body position; in future human studies, this might influence these measurements because off airway closure and airway secretions. Furthermore, we did use PEEP steps in a nonrandomized order which could influence results; if future PEEP titration studies would be performed, this should be a consideration.

\section{Conclusions}

Our study has shown that ventilatory inhomogeneity can be assessed with precision, using oxygen washout curves measured with the LUFU equipment without the need for external tracer gases. The moment ratio and LCI index appeared to be the most reproducible for integration with oxygen washout curves. Studies are under way to evaluate the indexes in critically ill patients. 


\section{Author details}

${ }^{1}$ Department of Intensive Care, Erasmus Medical Center, PO Box 2040, Rotterdam 3000 CA, the Netherlands.

${ }^{2}$ Department of Experimental Medical Instrumentation, Erasmus Medical Center, Rotterdam 3000 CA, the Netherlands.

${ }^{3}$ Department of Anaesthesiology, Erasmus Medical Center, Rotterdam 3000 CA, the Netherlands.

Received: 3 January 2014 Accepted: 23 March 2014

Published: 16 April 2014

\section{References}

1. Ricard JD, Dreyfuss D, Saumon G (2002) Ventilator-induced lung injury. Curr Opin Crit Care 8:12-20

2. Mead J, Takishima T, Leith D (1970) Stress distribution in lungs: a model of pulmonary elasticity. J Appl Physiol 28:596-608

3. Rausch SM, Haberthur D, Stampanoni M, Schittny JC, Wall WA (2011) Local strain distribution in real threedimensional alveolar geometries. Ann Biomed Eng 39:2835-2843

4. Riedel T, Kyburz M, Latzin P, Thamrin C, Frey U (2009) Regional and overall ventilation inhomogeneities in preterm and term-born infants. Intensive Care Med 35:144-151

5. Schibler A, Henning R (2002) Positive end-expiratory pressure and ventilation inhomogeneity in mechanically ventilated children. Pediatr Crit Care Med 3:124-128

6. Huygen PE, Feenstra BW, Holland WP, Ince C, Stam H, Bruining HA (1990) Design and validation of an indicator gas injector for multiple gas washout tests in mechanically ventilated patients. Crit Care Med 18:754-759

7. Huygen PE, Gultuna I, Ince C, Zwart A, Bogaard JM, Feenstra BW, Bruining HA (1993) A new ventilation inhomogeneity index from multiple breath indicator gas washout tests in mechanically ventilated patients. Crit Care Med 21:1149-1158

8. Gültuna I, Huygen PE, Jabaaij C, Holland WP, Ince C, Bruining HA (1992) A simple device to inject indicator gas for wash-out tests during mechanical ventilation. Intensive Care Med 18:304-308

9. Maisch S, Boehm SH, Weismann D, Reissmann H, Beckmann M, Fuellekrug B, Meyer A, Schulte Am EJ (2007) Determination of functional residual capacity by oxygen washin-washout: a validation study. Intensive Care Med 33:912-916

10. Bikker IG, van Bommel J, Dos Reis MD, Bakker J, Gommers D (2008) End-expiratory lung volume during mechanica ventilation: a comparison to reference values and the effect of PEEP in ICU patients with different lung conditions. Crit Care 12:R145

11. Larsson A, Jonmarker C, Werner O (1988) Ventilation inhomogeneity during controlled ventilation. Which index should be used? J Appl Physiol 65:2030-2039

12. Weismann D, Reissmann H, Maisch S, Fullekrug B, Schulte J (2006) Monitoring of functional residual capacity by an oxygen washin/washout; technical description and evaluation. J Clin Monit Comput 20:251-260

13. Horsley A (2009) Lung clearance index in the assessment of airways disease. Respir Med 103:793-799

14. Edelman NH, Mittman C, Norris AH, Shock NW (1968) Effects of respiratory pattern on age differences in ventilation uniformity. J Appl Physiol 24:49-53

15. Becklake MR (1952) A new index of the intrapulmonary mixture of inspired air. Thorax 7:111-116

16. Cumming G, Guyatt AR (1982) Alveolar gas mixing efficiency in the human lung. Clin Sci (Lond) 62:541-547

17. Saidel GM, Salmon RB, Chester EH (1975) Moment analysis of multibreath lung washout. J Appl Physio 38:328-334

18. Patroniti N, Saini M, Zanella A, Weismann D, Isgro S, Bellani G, Foti G, Pesenti A (2008) Measurement of endexpiratory lung volume by oxygen washin-washout in controlled and assisted mechanically ventilated patients. Intensive Care Med 34:2235-2240

19. Grotjohan HP, van der Heijde RM, Jansen JR, Wagenvoort CA, Versprille A (1996) A stable model of respiratory distress by small injections of oleic acid in pigs. Intensive Care Med 22:336-344

20. Wauer HJ, Lorenz BA, Kox WJ (1998) FRC measurement in intensive care patients. A definition of standards. Anaesthesist 47:844-855

21. Robinson PD, Latzin P, Verbanck S, Hall GL, Horsley A, Gappa M, Thamrin C, Arets HG, Aurora P, Fuchs SI, King GG, Lum S, Macleod K, Paiva M, Pillow JJ, Ranganathan S, Ratjen F, Singer F, Sonnappa S, Stocks J, Subbarao P, Thompson BR, Gustafsson PM (2013) Consensus statement for inert gas washout measurement using multiple- and single- breath tests. Eur Respir J 41:507-522, 10.1183/09031936.00069712 [doi]

22. Saidel GM, Saniie J, Chester EH (1980) Lung washout during spontaneous breathing: parameter estimation with a time-varying model. Comput Biomed Res 13:446-457

23. Mitchell RR, Wilson RM, Sierra D (1986) ICU monitoring of ventilation distribution. Int J Clin Monit Comput 2:199-206

24. Tsang JY, Emery MJ, Hlastala MP (1997) Ventilation inhomogeneity in oleic acid-induced pulmonary edema. J Appl Physiol 82:1040-1045

25. Lumb AB (2005) Nunn's applied respiratory physiology. Elsevier, Philadelphia

doi:10.1186/2197-425X-2-14

Cite this article as: Bikker et al:: Assessment of ventilation inhomogeneity during mechanical ventilation using a rapid-response oxygen sensor-based oxygen washout method. Intensive Care Medicine Experimental 2014 2:14. 